\title{
CAMBIOS Y PERMANENCIAS EN LAS FAMILIAS CON PADRES MIGRANTES RETORNADOS A ANTIOQUIA Y EL EJE CAFETERO COLOMBIANO
}

\section{CHANGES AND CONTINUITIES IN FAMILIES WITH MIGRANT PARENTS WHO HAVE RETURNED TO ANTIOQUIA AND THE COLOMBIAN COFFEE- GROWING REGION}

Jair Eduardo Restrepo PIneda*

Resumen: El artículo realiza una aproximación a las familias inmersas en un proceso de migración de retorno internacional de sus padres o madres, permitiendo visibilizar las modificaciones y la reconfiguración de sus dinámicas y cómo estas afectan las relaciones, la convivencia y el funcionamiento de la vida familiar. Aspectos tales como la autoridad y la toma de decisiones, los roles de género y la proveeduría económica son los que evidencian mayores cambios para todos los miembros de la unidad familiar. En cuanto a las permanencias se destaca el hecho de que el rol del cuidador sigue estando vinculado de manera inherente a la mujer. El retorno es un proceso dinámico en el cual la comunicación, el cuidado y la proveeduría económica preparan el camino del migrante para su reincorporación a la vida familiar.

Palabras clave: migración de retorno, dinámicas familiares, relación padre-hijo, Colombia.

Docente e investigador del programa de Trabajo Social de la Facultad de Ciencias Humanas y Sociales de la Corporación Universitaria Minuto de Dios UNIMINUTO - Sede Bello. jair.restrepo@uniminuto.edu Este artículo es producto de la investigación: "Familias migrantes retornadas a Antioquia y en Eje Cafetero", la cual fue financiada por UNIMINUTO -Sede Bello. 


\begin{abstract}
This article carries out an approach to the families immersed in a migratory process of international return of their fathers and mothers, allowing to make visible the modification and reconfiguration of their dynamics and how these affect the relations, cohabitation and functioning of family life. Aspects such as authority and decision-making, gender roles and economic supply are the ones that demonstrate greater changes for every member of the family unit. As regards the continuities, it is highlighted the fact that the carer role is still being inherently bound to the women. The return is a dynamic process in which communication, care and economic supply prepare the migrants' way towards their reincorporation into family life.
\end{abstract}

Keywords: return migration, family dynamics, parent-child relationship, Colombia.

\title{
1. INTRODUCCIÓN
}

La migración internacional no es un proceso reciente, su importancia actual radica en la profunda conexión con los procesos de globalización que se viven a escala mundial. Según datos de la Organización de las Naciones Unidas "en 2013, 232 millones de personas, o sea $3,2 \%$ de la población mundial, eran migrantes internacionales, a diferencia de los 175 millones registrados en 2000 y 154 millones en 1990" (ONU, 2013:1). Colombia no es ajena a esta situación, según datos del Departamento Administrativo Nacional de Estadística de Colombia (DANE), a partir del censo de 2005, se calculó que el número de colombianos en el exterior es de 3.378.345. El Ministerio de Relaciones Exteriores (2012), por su parte, estima una cifra aproximada a los 4.700.000, lo cual confirma el dato ofrecido por el DANE. Las estimaciones realizadas por el DANE referidas a los principales países de destino de los migrantes afirman que el 34,6\% se encuentra en Estados Unidos, el 23,1\% en España, el 20\% en la República Bolivariana de Venezuela, el 3,1\% en Ecuador, y el 2\% en Canadá.

Los departamentos de Antioquia y del Eje Cafetero (Caldas, Quindío y Risaralda) presentan uno de los índices más altos de emigración del país, convirtiéndose en un determinante para las nuevas dinámicas familiares. Es así, como el flujo migratorio colombiano está concentrado en cinco departamentos (que a su vez son los de mayor concentración de población). Éstos son: el Valle del 
Cauca con una tasa del 23,1\% de migrantes internacionales, Bogotá D. C. con el 17,6\%, Antioquia con el 13,7\%, Risaralda con el 6,9\% y Atlántico con el 5,8\% (DANE, 2005).

En el año 2010 se realiza la presentación del documento "Panorama del retorno reciente de migrantes internacionales a Colombia", dicho documento sostiene que "el retorno es un hecho de magnitud significativa y de importancia permanente en la definición de políticas y en la gestión migratoria, especialmente para los países de origen que deben enfrentarse al tema del reintegro social y económico de quienes regresan. En Colombia el tema ha sido poco estudiado" (Mejía, 2010:2). Este mismo documento realiza algunos cálculos en los cuales se establece que para el año 2005 el retorno de migrantes internacionales a Colombia se ubicaba en el orden de las 31.572 personas. Hacia el 2009, el volumen acumulado de retornados al país ascendía a los 420.000¹ (Mejía, 2010:8).

La Organización Internacional de las Migraciones (OIM) presenta en el año 2015 el estudio "El retorno voluntario desde España, estudios de caso: Bolivia, Colombia y Paraguay", el cual tiene como objetivo "conocer en profundidad el perfil, la percepción y las experiencias del retorno por parte de los beneficiarios del Programa de Retorno Voluntario" (Mejía et al., 2015:6). Las entrevistas realizadas en Colombia en el marco de esta investigación fueron 21; de ellas 11 se realizaron en el departamento del Valle, 3 en Risaralda, 2 en Antioquia, 2 en el Atlántico, 2 en Cundinamarca y finalmente 1 en el Norte de Santander (Mejía et al., 2015). Dichas entrevistas dan cuenta de las características del proceso migratorio, las motivaciones para el retorno y las intenciones de re-emigrar por parte de los entrevistados, sin embargo, no abordan las implicaciones que el retorno reviste en las dinámicas y las estructuras familiares.

\section{MARCO TEÓRICO}

Para abordar el tema de la migración de retorno Cassarino (2004) plantea cinco enfoques teóricos, estos son: la economía neoclásica,

1 Valor consistente con el que se obtendría extrapolando a todo el país y con la consideración de los sesgos presentes en la muestra, el porcentaje de hogares con retornados (5.5\%) y el promedio de ellos por hogar (1.21) arrojados por de la primera fase de la encuesta ENMIR1 $(n=75.852)$. 
la nueva economía de la migración laboral, el enfoque estructural, la perspectiva transnacional y la teoría de las redes sociales. Desde el enfoque neoclásico el retorno se fundamenta en las diferencias salariales entre los países emisores y receptores de migrantes, de manera tal que el retorno se verá condicionado por el cumplimiento de las expectativas financieras que le permitan asegurar el sostenimiento de la familia o en su defecto la reagrupación familiar. Por su parte, desde el enfoque de la nueva económica la migración de retorno se entiende como la consecuencia lógica de un cálculo estratégico, y como resultado de que los objetivos de la migración fueron obtenidos exitosamente (Stark, 1991). De otro lado, el enfoque estructural establece una vinculación entre las condiciones del país de origen y las expectativas del individuo. En este sentido, no solo las habilidades adquiridas o el capital económico acumulado por el migrante tienen un papel importante en la experiencia del retorno, sino que además juegan un rol significativo el contexto de origen de los migrantes y sus relaciones de poder, valores y tradiciones, es decir, los factores sociales e institucionales del país de origen. Desde la perspectiva transnacional, Alfaro e Izaguirre (2003:5) manifiestan que "el retorno ocurriría cuando el migrante ha acumulado suficiente cantidad de recursos financieros o informativos, y cuando las condiciones en el lugar de origen aparecen ser favorables. El proceso no está exento de tensiones, y quienes retornan pueden sentir marginalización, pero tratarán de renegociar sus lugares en la sociedad movilizando recursos que derivan de un común de atributos compartidos" Finalmente, el enfoque de redes sociales pone de manifiesto como una estructura social y su configuración a través de los vínculos es primordial para comprender como se crean y se mantienen las relaciones transfronterizas, sostenidas en los vínculos interpersonales duraderos, así como en el intercambio constante de bienes tanto tangibles como intangibles entre los involucrados.

Estos cinco enfoques sobre el retorno ponen de manifiesto la importancia de considerar no solo los determinantes económicos de este proceso, sino también los factores personales, familiares, sociales, culturales, institucionales, entre otros que contextualizan la decisión de retornar.

Por otro lado, se reconoce a la familia como una realidad social en permanente y constante movimiento, así "los cambios y transformaciones que le son inherentes se encuentran entretejidos 
con los procesos económicos, sociales, políticos y culturales que acompañan la sociedad a la cual pertenece." (Palacio, 2006: 452).

En todo proceso migratorio que implique el desplazamiento de los padres se genera una modificación alrededor de los vínculos afectivos y de cuidado. Esta situación es más evidente en las relaciones familiares y de pareja ya que se produce un "sentido de pérdida ambigua, ocasionada por el desarraigo físico, social y cultural, que manifiestan todas las personas, con más o menos intensidad, que han pasado por un proceso de migración" (Delatte et al., 2009:6).

Es así como los procesos migratorios internacionales tienen entre sus principales consecuencias las modificaciones en las dinámicas familiares que afectan las normas, las reglas, los límites, las jerarquías y los roles entre los miembros de la unidad familiar, de igual manera cuando este proceso llega a la etapa de retorno todas estas dinámicas deben reestructurarse para hacer frente a una nueva realidad (González y Restrepo, 2010; Ghiso et al., 2009). Además, cuando el padre o la madre han tomado la decisión de retornar, surge una nueva cuestión: ¿Qué tipo de cambios y permanencias se establecen en los vínculos e interacciones familiares a partir de los procesos de migración y de retorno?

Un autor como Hondagneu (1994) propone tres procesos migratorios familiares; a saber, la migración gradual de la familia, la migración unificada y la migración independiente. Gonzalez de Rocha (2002) habla sobre los cambios producidos en la familia a raíz de la migración, al igual que Mummert (1996) quien aborda los cambios en los roles de las mujeres inmersas en un proceso migratorio internacional, y Kanaiaupuni (1999) quien analiza las relaciones de género en torno a las expectativas migratorias y los patrones de estas. Todos estos autores coinciden en afirmar que las migraciones han contribuido a la emergencia de nuevas formas de organización familiar. El estudio de la familia desde la perspectiva transnacional permite analizar la interrelación entre las estrategias económicas y las de reproducción social que determinan los ajustes, las formas y los significados que se establecen entre los migrantes y aquellas personas que permanecen en origen, logrando con ello mantener los vínculos de afecto y cuidado en un contexto de dependencia.

Para Rivas (2008) las familias transnacionales son construcciones sociales o comunidades imaginadas en medio de lazos emocionales y económicos, las cuales se crean y recrean a través de relaciones 
que traspasan las distancias geográficas para darle un sentido a la vida familiar. Sin embargo, esta nueva realidad genera cambios en el transcurrir transnacional, generando planes y proyectos futuros, los cuales son concebidos alrededor de volver a estar juntos, bien sea a través del retorno o de la reunificación familiar (Nyberg, 2008).

Las relaciones y las dinámicas familiares se expresan y mantienen a través de los lazos económicos como las remesas o las conversaciones frecuentes a través de los medios virtuales y de comunicación. Todas estas interacciones son atravesadas por los afectos o sentimientos de amor, deseos de estar juntos y a la vez duelos ante las separaciones que la residencia en otro país genera para los y las migrantes, y las relaciones vinculantes establecidas con el país de origen (Puyana et al., 2010).

Por su parte, Alfaro e Izaguirre (2003) sostienen que la migración de retorno genera en las relaciones familiares y personales un proceso de ajuste a una nueva dinámica social. Dicho ajuste se manifiesta en la readaptación y la reincorporación del retornado a su núcleo familiar, así como en la negociación de expectativas o de conflictos entre ambas partes. Para Guarnizo (2006) se presentan al menos cinco señales de transformaciones en la familia cuando viven una experiencia migratoria. La primera de ellas, la alteración de patrones sociales de relación desde la estructura de la familia y de las relaciones de género; en segundo lugar, los cambios de las reglas familiares; tercero, la alteración de ordenamientos de autoridad y poder cuando la jefatura se traslada al exterior; cuarto, el vacío de autoridad y afecto no pueden ser llenado totalmente por las comunicaciones a larga distancia; por último, la movilización de apoyo a parientes en el cuidado y atención a los hijos.

El proceso de retorno origina dentro de las familias una nueva dinámica que demanda generar estrategias para adaptarse a este evento en el cual el padre o la madre vienen a ocupar un lugar dentro de esta después de un largo periodo de ausencia. Todo esto supone asumir nuevos roles y situaciones para los cuales el retornado, así como los miembros de su familia generalmente no están preparados.

\section{METODOLOGÍA}

Con la finalidad de alcanzar el objetivo del estudio que consistió en entender la manera cómo las modificaciones producto de la 
migración de retorno afectan la convivencia y el funcionamiento de la vida familiar y qué estrategias adoptan las familias para hacer frente a esta nueva situación, se utilizó el enfoque de investigación cualitativo de tipo descriptivo. Se inició con la aplicación de una entrevista semi-estructurada destinada al padre retornado, a por lo menos uno de los hijos y al cuidador en el caso que este estuviera presente en la vivienda. Las entrevistas se organizan en categorías de análisis que enmarcan todo el proceso migratorio, es decir, antes de dar inicio a este, durante el tiempo de residencia del migrante el país de destino y finalmente su retorno al país de origen. En total se intervinieron 11 familias, en las cuales se realizaron 24 entrevistas, 2 correspondientes a cuidadores, 11 a hijos ( 8 varones y 3 mujeres), 5 a padres y 6 a madres migrantes retornadas; todos ellos residentes en los departamentos de Antioquia y el Eje Cafetero de Colombia.

La entrevista se realizó a las familias que cumplieron los siguientes criterios de inclusión: El padre o la madre hubieran retornado de cualquier país extranjero a hacer parte de su familia en Colombia; la familia a la que se retornó se encuentra residiendo en los departamentos de Antioquia y el Eje Cafetero (Caldas, Quindío o Risaralda); los hijos de los padres retornados debieron permanecer en Colombia durante el proceso de migración parental; y la experiencia migratoria del entrevistado debería ser igual o superior a un año.

Los datos de contacto de las familias se obtuvieron de diferentes fuentes oficiales, entre ellas el Subprograma de Migrantes de la Alcaldía Municipal de Pereira (Risaralda), el Programa de Migrantes de la Alcaldía Municipal de Dosquebradas (Risaralda), además de la Organización Internacional de las Migraciones (OIM). Los padres firmaron un consentimiento informado en el cual permitían que sus hijos menores de edad participaran en la investigación, los nombres utilizados en el artículo son ficticios con el fin de proteger la privacidad de las familias.

\section{RESULTADOS}

La edad promedio de los migrantes retornados es de 46 años, en rangos que van desde los 34 a los 56 años. Es decir, todos se encuentran en edad de trabajar. Por su parte, los hijos entrevistados tienen en promedio 22 años de edad; de estos, 9 corresponden a 
población joven (entre los 14 y 28 años) y solo 2 son mayores de 29 años. Los cuidadores que aún hacen parte de la familia del migrante retornado y pudieron ser entrevistados tienen en promedio 45 años de edad. Los cuidadores entrevistados fueron 1 varón y 1 mujer y sus relaciones de parentesco eran tío y abuela, respectivamente.

El nivel educativo de los entrevistados demostró ser bajo. Sólo 2 alcanzaron el grado de técnica, igual número finalizó el bachillerato y la mayoría de ellos solo tienen la educación primaria concluida, siendo las mujeres las que mejor nivel educativo presentan. Estos datos demostrarían lo que sostienen autores como Borjas \& Bratsberg (1996) y Mejía (2010) sobre el proceso de selectividad en el retorno, es decir, mientras migran las personas con mejores niveles educativos aquellos que retornan suelen ser los menos educados. Finalmente, en cuanto al país de retorno, 6 de los entrevistados proceden de España, 2 de Japón, 1 de Bolivia, 1 de Ecuador y 1 de Venezuela.

En el cuadro 1. Características generales de las familias retornadas entrevistadas, se presenta la información relativa a estas que permite conocer el contexto en el cual se dio la migración y algunos datos importantes para comprender los cambios que sufrieron las dinámicas y las estructuras familiares.

El tiempo promedio de permanencia de los padres en el exterior es de 10 años. La edad media de los hijos en el momento de la migración del padre o madre es de 11 años. Frente a las motivaciones del retorno, 5 de los entrevistados debieron retornar por falta o vencimiento de documentos para residir legalmente en el país de destino, 3 por motivos económicos o laborales y 3 por causas familiares. Es decir, de los 11 entrevistados, 8 debieron retornar por causas ajenas a su voluntad relacionadas con el acceso al mercado laboral. Por tanto, "cuando la coyuntura económica en el país receptor contrae drásticamente el empleo, los migrantes acaban por retornar a su país de origen ante la falta de puestos laborales" (Mestries, 2013:172), tal situación se pudo presentar durante la crisis económica española que se inició en el año 2008 y tuvo fuertes repercusiones sobre los migrantes. 


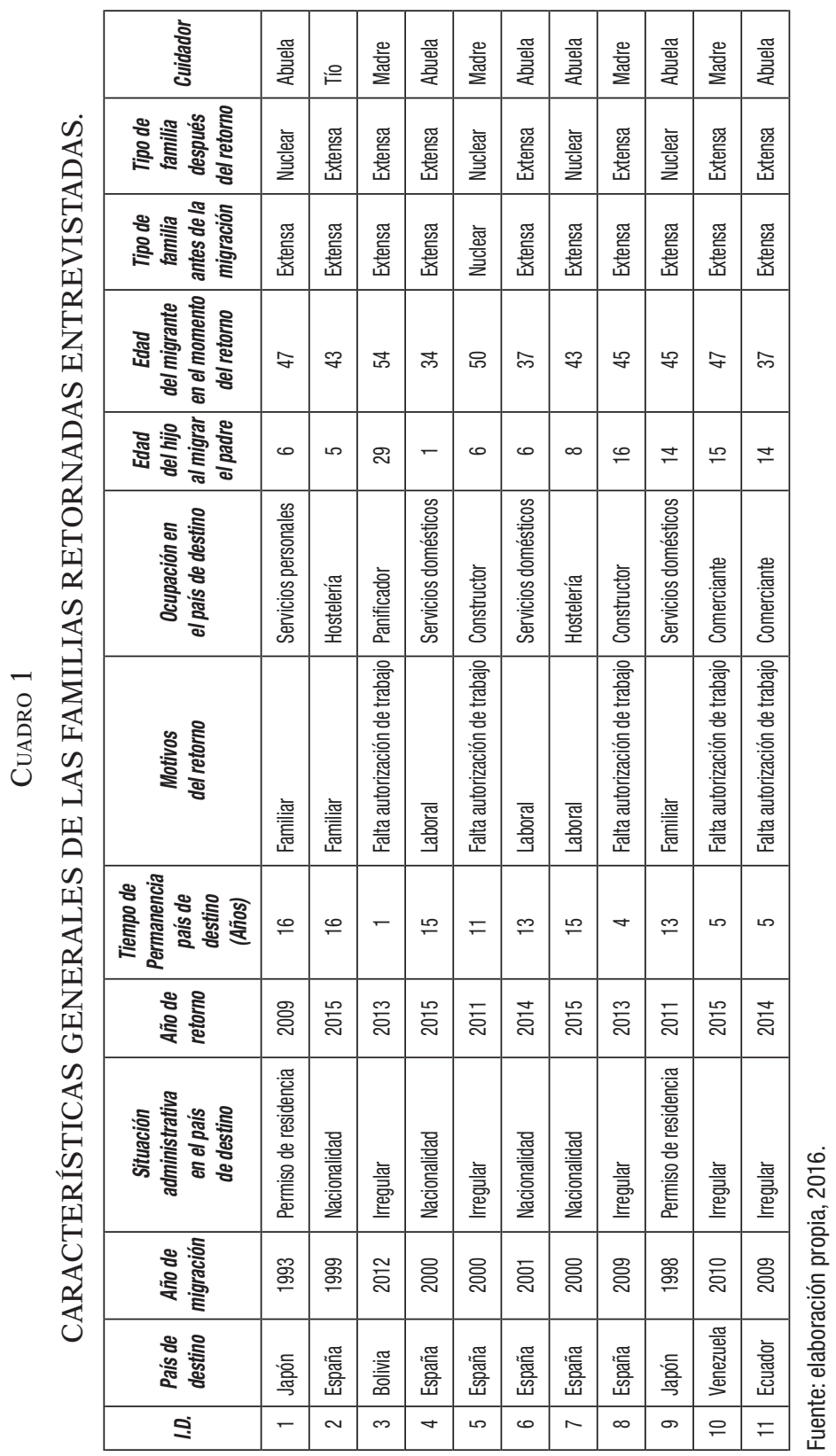




\section{ESTRUCTURA FAMILIAR}

En las 11 familias entrevistadas tanto la migración como el proceso de retorno implicó cambios en la conformación de estas en cuanto a la entrada y salida de nuevos integrantes, así como en los roles y las responsabilidades de sus miembros.

En los casos estudiados, los hijos de los migrantes pasaron al cuidado de terceras personas mientras duró la migración de sus padres, en ninguno de los casos esto significó su incorporación a otras unidades familiares, ya que de los 11 casos estudiados en 10 el cuidador residía con ellos aun antes de dar inicio el proceso migratorio:

"Sebastián nació en mi casa antes de que mi hermana se fuera, o sea que nunca fue un nuevo miembro, siempre estuvo ahi" (Andrés, 39 años, hermano de migrante retornado, Pereira).

Las características de estas familias responden a particularidades culturales de la región antioqueña donde "la familia presenta un marcado sabor matriarcal, y fuertes nexos familiares en la unidad extensa unilineal uterina" (Gutiérrez de Pineda, 1975:33). Es decir, alrededor de la abuela materna se centralizan las relaciones y dinámicas de los núcleos familiares generando una red de solidaridad y cooperación que sirve de sustento a todos los individuos que conforman la familia extensa, esto explicaría porque mayoritariamente son las abuelas maternas las encargadas del cuidado de los hijos del migrante y no otros familiares.

Por otra parte, en el momento del retorno se presenta un cambio en la estructura de la familia, ya que de las 11 familias entrevistadas, 10 eran extensas y 1 nuclear antes del inicio del proceso migratorio, después del retorno 7 siguen siendo extensas mientras que las 4 restantes pasan a ser nucleares. El cambio en la configuración de familias extensas a nucleares se debe entre otras razones al mejoramiento de sus condiciones económicas que les permiten el acceso a una vivienda propia logrando independizarse, ya que en la mayor parte de los casos la vivienda pertenecía a los padres del migrante. Además, se observa que aquellas familias estudiadas que pasan de extensas a nucleares provienen de países asiáticos y europeos donde la estructura familiar que prevalece es la nuclear o conyugal, algunos de los migrantes retornados regresan con sus parejas de estas nacionalidades y dan continuidad a sus vidas en la mismas condiciones familiares que tenían en el país de destino. 


\section{RELACIONES FAMILIARES}

Tanto el proceso de migración como el de retorno tienen implicaciones directas e indirectas en los miembros de la familia, ya que modifican la cotidianidad y por lo tanto las dinámicas, las funciones, los vínculos y los roles dentro de esta. Además, se debe tener en cuenta que "las relaciones son establecidas y avaladas desde lo cultural, lo social y lo familiar, no tienen la misma intensidad entre todos los integrantes y dependen del rol que desempeñe y de los vínculos que se establezcan" (Ghiso et al., 2009a:31).

El retorno del migrante implicará que las relaciones familiares se reconfiguran en el escenario de la cotidianidad, donde el contacto y encuentro permanente entre sus miembros construyen y consolidan sus relaciones y dinámicas. Así, se establecen vínculos afectivos, se imponen normas y pautas de comportamiento y se promueve el respeto entre sus miembros, es decir, "las familias organizan la vida cotidiana alrededor de una serie de actividades que promueven su identidad" (Migliorini et al., 2011:185).

Aspectos tales como la proveeduría económica y la comunicación determinan de qué manera se relacionarán los miembros de la unidad familiar y qué estrategia afrontarán para mantener los vínculos afectivos y de cuidado. Los cambios en las relaciones familiares pueden ser de diversos tipos, ya sean en su estructura familiar, en los roles, en el poder o la autoridad. Por otra parte, el retorno permite el encuentro entre experiencias que implican "cambios, transformaciones y readecuaciones en las relaciones,... haciendo que... estas... se reorganicen o se implementen nuevos mecanismos para el funcionamiento cotidiano" (Aznar, 2009:21).

\section{RELACIONES DE CUIDADO}

En los casos en los cuales la tarea de cuidador fue asumida por otro familiar diferente a la pareja del migrante retornado - en 1 de los casos el tío y en 6 la abuela-se presentaron 2 situaciones después del retorno: la primera que el cuidador permanezca haciendo parte de la unidad familiar y la segunda que por el contrario deje de ser parte de esta. En el primero de los casos, se observa que ni antes ni después de la migración la relación de cuidado cambia de manera 
considerable, y por lo tanto "cuando los migrantes pertenecen a una familia numerosa y extensa, las relaciones no se ven tan afectadas por la migración, contrario a lo que ocurre cuando se hace parte de una familia pequeña y nuclear" (Ghiso et al., 2009a:33). En palabras de un hijo de migrante retornado:

"Mi abuela, porque yo soy como un hijo para ella, porque desde chiquito ha vivido conmigo, me vio nacer, antes de irse mi mamá trabajaba y yo estaba todo el día con mi abuelo y con mi abuela" (Sebastián, 24 años, hijo de migrante retornado).

Cuando se encuentran presentes los 3 actores del proceso de retorno, padre o madre, hijos y cuidador, las relaciones se mantienen estables y cordiales. Los hijos de los migrantes retornados ven en sus cuidadores una figura de respeto, además se hicieron evidentes las muestras de afecto y cariño hacia estos durante toda la realización de las entrevistas. De manera general, las abuelas estuvieron a favor de la decisión migratoria de sus hijos y el hecho de quedarse a cargo de sus nietos era considerado una forma de contribuir en este proceso que además involucraba un deber como madres.

En el segundo caso, cuando los cuidadores ya no hacen parte de la familia del retornado, se puede presentar un deterioro en la relación entre el hijo y el cuidador debido a problemas en la convivencia y a los tratos que se dieron durante el tiempo que duró la migración de la madre. Un entrevistado afirma:

"No tengo contacto con mi abuela y eso que vivimos a 5 calles. A pesar de todo es una buena persona. Hay que saber perdonar" (Jorge, 33 años, hijo de migrante retornado, Dosquebradas).

En los casos en los cuales el papel de cuidador lo asume el padre o la madre que no migró las relaciones se mantuvieron estables y el papel de este fue fundamental para asegurar los vínculos afectivos y emocionales del migrante con sus hijos, un padre retornado manifiesta:

"Las madres de mis hijos los educaron muy bien y ellas les decían a mis hijos que en esto viene el papá y ustedes saben que él los quiere mucho. Además, los niños constantemente hablaban conmigo por teléfono" (Juan, 54 años, migrante retornado, Pereira). 
Así, el progenitor que no migró se encarga de recordar a sus hijos la existencia no solo física sino emocional de la otra figura paterna. Esto permitió que en el momento del retorno los hijos valoraran y reconocieran a su padre como parte esencial y funcional de la unidad familiar. En algunos de los casos estudiados, las relaciones de cuidado recuperan la dinámica que tenían antes de la migración, los padres ocupan sus lugares como los encargados se asegurar el bienestar de sus hijos y en algunos casos de los demás miembros de la familia extensa.

Finalmente, en el caso de los hijos mayores de edad las relaciones de cuidado son desplazadas por aspectos relativos a la proveeduría económica de la familia, así:

"Pues había mucho conflicto porque no había mucho dinero, no había como subsanar los gastos de la casa, entonces habia mucho conflicto por eso" (Diana, 30 años, hija de migrante retornado, Dosquebradas).

Esta situación no cambia con el retorno, ya que los problemas económicos que sufre la familia se ven incrementados por la disminución de los recursos provenientes de las remesas y de manera general porque el migrante retornado no accede fácilmente al mercado laboral de su país de origen.

\section{RELACIONES DE AUTORIDAD Y PAUTAS DE COMPORTAMIENTO}

En el momento en el cual el padre o la madre migrante se incorpora a su unidad familiar se reconfiguran de nuevo los roles y se asumen las responsabilidades que habían sido delegadas en otros miembros de la familia, especialmente en los temas de cuidado y autoridad. Sin embargo, en todos los casos, antes del retorno estos roles eran reforzados permanentemente por el padre que no migró o por el cuidador. El siguiente testimonio así lo evidencia:

"Para la decisión cuando ellos estaban conmigo los permisos a la calle, sus trabajos y todo eso, todo eso la tomaba yo acá, pero también le pedía consentimiento a ellos (migrantes) que opinaban ellos del permiso que yo daba o la decisión que yo tomaba" (Flor, 51 años, madre de migrante retornado, Pereira). 
Durante el proceso migratorio se identificaron dos tipos de estrategias de autoridad; la primera de ellas, la estrategia de autoridad directa y, la segunda, la de intermediación a cargo del cuidador (González y Restrepo, 2010). Cuando el migrante retorna asume de nuevo como figura de autoridad dentro de la familia, sin embargo, esta ha variado en cuanto a sus características, ya que no solo está en función de la proveeduría económica y en manos del varón, sino que los conocimientos y saberes culturales que el migrante trae en forma de remesas sociales (Di Leonardo, 1992; Bryceson \& Vuroela, 2002) modificarán de manera considerable la forma en la cual se relaciona la familia y las dinámicas que establece.

"Se habla entre todos y se llega a una conclusión pero siempre se conversa entre todos" (Flor, 51 años, madre de migrante retornado, Pereira, septiembre de 2015).

Es así como algunos migrantes dejan de lado la autoridad centrada en ellos mismos, que se podría definir como un estilo autoritario, y pasan a un proceso de conciliación al interior de la familia donde todos los miembros participan, incluyendo a los cuidadores, situación que pone de manifiesto que estos no son excluidos de las dinámicas familiares posteriores al retorno. Este nuevo estilo de autoridad se le puede denominar como democrático, en palabras de los entrevistados:

"Aqui la autoridad la tenemos todos, mi esposo y yo, nosotros hablamos con los muchachos de las cosas y tomamos las decisiones, pero siempre hablamos, porque ellos tiene que entender las cosas" (Janeth, 43 años, migrante retornado, Medellin).

Estos cambios generan en la familia una transformación en sus relaciones ya que sus miembros empiezan a apropiarse de nuevas prácticas, de una nueva manera de interactuar y de convivir que llega a los miembros de las familias gracias a la experiencia del migrante y a los aprendizajes sociales y culturales que este trae en forma de remesas sociales. Un migrante retornado manifiesta:

"El cambio fue total, yo por allá en ese país aprendí a vivir solo, porque me tocó vivir solo y yo le digo antes de irme yo de este país a ser inmigrante, yo no sabía hacer una agua de panela, a mí se me quemaba un agua en el sartén..., entonces me toco aprender a hacer alimentos, me toco aprender a hacer limpieza en la casa, todo" (Juan, 54 años, migrante retornado, Pereira). 
Es así como los padres participan de manera activa y continua en labores que antes eran delegadas única y exclusivamente a las mujeres y esto se convierte en un referente para sus hijos, quienes finalmente interiorizaran relaciones más equitativas entre varones y mujeres.

Por su parte, las pautas de comportamiento que en algunos casos han sido modificadas por los cuidadores durante la ausencia de los padres se mantienen después de su retorno, dichas cambios se refieren principalmente a las responsabilidades que deben ser asumidas por los hijos dentro de la familia. Así:

"La abuela puso las normas, ya uno tenía que hacer la habitación de uno, ser más ordenados" (Anderson, 14 años, hijo de migrante retornado, Pereira).

Las pautas y normas de comportamiento están muy relacionadas con la edad de los hijos del migrante, ya que, como lo manifiestan algunos de los entrevistados, estas son asumidas de manera más fácil en edades tempranas que cuando son adolescentes o jóvenes. Sin embargo, en las narraciones tanto de los padres como de los cuidadores y de los hijos se mantiene una continuidad en las normas durante la migración y el retorno.

Estas normas giran en torno al desempeño académico, al cumplimiento de responsabilidades de tipo doméstico y al control de salidas y llegadas a la vivienda (Puyana, 2003). Todas ellas relacionadas con las edades de los hijos pero no divididas por géneros. El hecho de que los padres retornados exijan por igual a los varones como a las mujeres participar en las tareas domesticas y cumplir con los horarios de entrada y salida de la vivienda, evidencia un avance hacia la equidad de género que pudo haber sido aprendido en su experiencia como migrante.

Además, se da continuidad a las reglas implantadas por los padres antes de la migración, aunque estas se hayan modificaron durante la ausencia de los padres haciéndose más flexibles e incorporándose nuevas normas, especialmente cuando quien estaba a cargo de los hijos eran los abuelos.

"Lo que pasa es que la abuela lo cuidaba y lo consiente mucho, aunque ya es un hombre, ella le daba mucha libertad y pues cuando volví quería hacer lo que él decía" (José, 47 años, migrante retornado, Medellín). 
Esta situación pudo haber originado algún tipo de conflicto entre los padres y los hijos, sin embargo, la cotidianidad y el establecimiento de nuevas tácticas redefinen las relaciones entre estos. Así, los padres al retornar implantan nuevas reglas para lograr el cumplimiento de la norma, esencialmente aquellas referidas a la prohibición o suspensión del uso y disfrute de ciertos bienes o servicios, tales como la telefonía celular, la internet, la televisión o las autorizaciones para salidas.

\section{RELACIONES DE AFECTIVIDAD Y COMUNICACIÓN}

Durante el proceso migratorio todas las familias entrevistadas se valieron de las llamadas Tecnologías de la Información y la Comunicación (TIC) para mantener el intercambio de información entre sus miembros. Estas herramientas posibilitaron además el aumento del capital emocional, referido a los sentimientos personales como los afectos, la confianza y el compromiso.

En 8 de las once familias entrevistadas, una vez el migrante retorna y se reincorpora a su familia, las expresiones de afecto aumentan y el dialogo entre los hijos y los padres también lo hace, tal y como se evidencia en este testimonio:

"Yo a veces lo veo en el cuarto y me entro al cuarto de él y le doy besitos, le digo que lo quiero, que tan lindo mi muchacho "(Sandra, 53 años, migrante retornado, Dosquebradas).

Durante el retorno las herramientas utilizadas para efectuar la comunicación cambian y las expresiones de afecto y el contacto personal aumentan, lo cual mejora y consolida los vínculos entre los miembros de la familia.

Así, 8 de los 10 padres entrevistados manifestaron que la comunicación con sus hijos ha mejorado con relación a la que tenían antes de migrar, ya que han observado mayores niveles de confianza en sus conversaciones. Además, las relaciones son más afectuosas con sus hijos y más cordiales con otros miembros de la familia. Los cambios en la comunicación que se evidencian entre los padres retornados y sus hijos están relacionados con el ciclo vital de estos, es decir con su crecimiento, ya que en la mayoría de los casos los hijos han quedado en la etapa de la niñez cuando el padre inició su proceso migratorio y al retornar estos se encuentran en la adolescencia o la juventud. 
La comunicación es un factor presente durante todas las etapas del hecho migratorio y al retornar los padres observan que ha cambiado no solo el tono de la comunicación, sino que el nivel de confianza ha aumentado y los temas que se abordan durante las charlas se han ampliado. Esta situación también tiene su origen en las nuevas percepciones y conocimientos que adquiere el migrante durante su estadía en el extranjero, que le permiten hablar más libremente con sus hijos de temas que inicialmente consideraban difíciles de tratar como la sexualidad, el consumo de sustancias psicoactivas, las responsabilidades frente a la familia y a sí mismos, entre otros:

"Antes para mí era muy difícil hablar con mi hijo pero uno ve muchas cosas por allá y hablamos y le aconsejo y él me cuenta sus cosas personales" (Carolina, 49 años, migrante retornado, Medellín).

Es indiscutible la importancia de la comunicación dentro de los miembros de la familia y especialmente entre los padres e hijos que les permitan recrear este vínculo.

\section{EL PAPEL DEL CUIDADOR}

Después del retorno del migrante la figura del cuidador no desaparece por completo, solo en aquellos casos en los cuales las familias se transforman de extensas a nucleares el cuidador pierde las funciones que le fueron delegadas, aunque su rol de tío, tía, abuelo o abuela continúan de forma permanente (Ghiso et al., 2009b).

"Pues bien, yo la voy bien con ella, yo a veces la llamo, a veces voy porque usted sabe que es la mamá y ella está muy enferma y uno de vez en cuando debe de ir a ver la mamá" (Sandra, 53 años, migrante retornado, Dosquebradas).

Cuando el cuidador sigue siendo parte de la familia del migrante retornado este no cumple una función pasiva; en algunos casos aporta económicamente al sostenimiento del la familia y en la mayoría de las situaciones toma parte en las discusiones y decisiones que se generan en torno al bienestar familiar. Además, el papel de los cuidadores es muy bien valorado por el migrante retornado ya que reconoce que sin su colaboración su salida del país no hubiera sido posible. En palabras del retornado: 
"Ni mamá nos ayudo mucho, es que si ella no se hubiera quedado con mis hijos yo no me voy, me quedo aqui con mis hijos" (Janeth, 43 años, migrante retornado, Medellin).

Estas situaciones ponen de manifiesto que las relaciones se reconfiguran ya que se vuelven más democráticas en cuanto a la participación de todos los miembros en la toma de decisiones, los aportes que estos hacen al sostenimiento familiar y a la consolidación de los lazos afectivos y de solidaridad dentro de la unidad familiar. Sin embargo, un aspecto que se mantiene constante dentro de estas familias es la responsabilidad que se le asigna a la mujer, ya sea madre, abuela o hija, de mantener y consolidar los lazos afectivos y de solidaridad familiar. Es así, como encontramos familias con padres retornados en las cuales las abuelas siguen haciéndose cargo de sus hijos, si bien estas participan de manera más activa en la toma de decisiones o en la proveeduría económica de la familia continúan cumpliendo un papel fundamentalmente de cuidadoras.

\section{PROVEEDURÍA ECONÓMICA}

La proveeduría económica es uno de los factores que más se ve afectado en el momento del retorno, especialmente en los casos en los cuales los procesos migratorios no fueron exitosos. Estas situaciones se afrontan de diversas formas, entre ellas a través de la solidaridad de los parientes que conforman la familia extensa. Así:

"Económicamente ha cambiado porque ya solo manda remesas mi papá y antes mandaba mi papá y mi mamá, ahora los gastos dependen de la abuela" (Anderson, 14 años, hijo de migrante retornado, Pereira).

Es importante anotar que las circunstancias económicas así como los demás asuntos de la familia son de conocimiento de todos sus miembros, incluyendo los hijos, esta situación refuerza la idea de un modelo de familia más democrático en el cual las cargas económicas se distribuyen de acuerdo a las posibilidades de cada integrante de la familia (Ghiso et al., 2009b). En otros casos, un familiar del retornado asume la proveeduría económica mientras que este puede encontrar un empleo. 
"Mi madre y yo, mi madre es pensionada y yo que trabajo y en este momento la madre le colabora al hijo para el sostenimiento... aporta un dinero al hogar solamente por el sostenimiento, es un apoyo no más, una colaboración" (Andrés, 39 años, hermano migrante retornado, Pereira).

Si bien, la proveeduría económica es un factor que condiciona la autoridad, en los casos de las familias con padres o madres retornados dicha autoridad se negocia entre sus miembros no solo teniendo en cuenta sus aportes económicos sino el papel que desempeñan dentro de la unidad familiar. De manera general, todas las familias manifiestan una disminución en los ingresos que llegan a la familia después del retorno, ya sea porque ambos padres estaban en el exterior y uno de los dos retornó reduciendo la cantidad de remesas que perciben.

O bien porque el dinero que ganan en Colombia no es comparable con aquel que recibían trabajando en países como España durante la época de crecimiento económico, es decir se presenta un crecimiento decreciente de la migración (Durand, 2004).

Además, el uso de las remesas por parte de las familias en el país de origen no es destinado a actividades de inversión productiva o de emprendimiento, sino al consumo. Esta situación se hace evidente en esta entrevista:

"Sí, sin duda subimos más de estrato, cambiamos de barrio, antes vivíamos por allá en Cuba, subimos al centro, cambio todo el amoblado de la casa, el salón, la nevera, la lavadora... todo ha cambiado para mejor" (Sebastián, 24 años, hijo de migrante retornado, Pereira).

Esto corroboraría que "las personas perceptoras de remesas destinan dichos recursos económicos a la compra de tierra, vivienda y otros activos reales no productivos, otra cantidad en educación y sanidad (Valero-Matas et al., 2014:33). Así, el uso de las remesas destinadas a la subsistencia familiar y al consumo dejando de lado la inversión productiva y el emprendimiento pueden generar en estas familias problemas relacionados con la proveeduría económica.Esta situación puede acarrear una dependencia económica familiar casi exclusiva de las remesas y en el momento del retorno la familia no contaría con otras fuentes de sostenimiento económico.Vale la pena mencionar que más de la mitad de los retornados entrevistados se en- 
cuentran desempleados y consideran que será muy difícil su vinculación laboral si se tienen en cuenta sus edades y su nivel de formación.

\section{ROLES FAMILIARES}

Las relaciones de poder dentro de la familia están marcadas por el género, el cual origina en su interior una estratificación que a su vez establece para sus miembros funciones determinadas por un estatus y un rol. Sin embargo, se pueden observar transformaciones en las relaciones de género dentro de las familias estudiadas, de manera más significativa cuando es el padre quien ha retornado, pues los relatos así lo plasman:

"Arregla la casa, hace de comer, me cuida a los abuelos, hace turnos en panadería donde lo llaman" (Diana, 30 años, hija de migrante retornado, Dosquebradas).

De esta manera, los padres asumen roles que social y culturalmente le habían sido delegados a la mujer, sin que ello signifique un detrimento en su autoridad familiar y su posición frente a los demás miembros de la familia. Por el contrario, los roles se reconfiguran permitiendo al retornado no solo asumir las responsabilidades que tenía antes de la migración, sino que además incorpora nuevos saberes, conocimientos y percepciones adquiridos en el país de destino, los cuales a través de la cotidianidad harán parte de las dinámicas familiares permitiendo construir relaciones más equitativas e igualitarias.

Estos cambios en los roles de género han llevado también a que emerjan madres e hijas que tras el proceso migratorio de sus parejas y padres hayan asumido nuevas expectativas de vida. Así, algunas de ellas se han hecho cargo en mayor medida de la proveeduría económica de su familia aun después del retorno de los varones o han asumido un papel central como figura de autoridad dentro de la unidad familiar.

Los cambios en los roles de género involucran a todos los miembros de la familia, madres, abuelas, hijos e hijas, quienes a través de la cotidianidad empiezan a interiorizar dichos conocimientos. 


\section{CONCLUSIONES}

En las transformaciones en los vínculos y las relaciones familiares se hace evidente un acercamiento más profundo entre los padres y los hijos después del retorno que se manifiesta en mayores expresiones de afecto, sin importar la edad del hijo. De igual manera sucede con la comunicación, la cual se hace más constante y con mayores niveles de confianza entre padres e hijos. Se debe decir que estos cambios se posibilitan gracias a la intervención del cuidador como soporte permanente del proceso migratorio y a las estrategias de comunicación de las cuales se valieron los padres durante su ausencia, además de los aprendizajes sociales y culturales que obtuvieron en los países de destino. Asimismo, las remesas se convierten en el medio a través del cual el padre puede expresar su preocupación, compromiso y respaldo a la unidad familiar. De alguna manera la ausencia física empieza a ser subsanada a través de las remesas. Todos estos procesos confirman los argumentos de autores como $\mathrm{Pa}-$ lacio (2006), Rivas (2008), Nyberg (2008) y Puyana (2010), quienes sostienen que las familias son estructuras dinámicas que responden a las nuevas demandas sociales, las cuales se articulan a través de lazos emocionales, de cuidado y de proveeduría económica, que no son ajenos a la migración de retorno.

Si bien es cierto que durante los primeros meses del proceso migratorio se crea un estado de crisis para toda la familia, también lo es que la reconfiguración de roles y responsabilidades dentro de esta ayudan a paliar los efectos negativos que se puedan generar con la partida del padre o la madre. En este proceso emerge el papel del cuidador como actor fundamental durante la migración, el cual, como se hizo evidente en las entrevistas, no desaparece de la vida familiar con el retorno del migrante, sino que adquiere - como se vio en algunos casos- un papel principal en las relaciones y dinámicas familiares. La vinculación de otros actores, como los cuidadores, que no solo aportan al bienestar de los hijos sino que además pueden ayudar económicamente al sostenimiento familiar, además de los aprendizajes que el migrante retornado trae en forma de remesas sociales, se convierten en los elementos que servirán para reconfigurar las dinámicas y relaciones familiares, las cuales resultan ser más equitativas, igualitarias y democráticas. Aspectos tales como la toma de decisiones, las responsabilidades y la proveeduría económica son los que evidencian mayores cambios 
para todos los miembros de la unidad familiar. Es así, como padres e hijos participan de forma más activa en las tareas domesticas. Las mujeres, ya sean estas madres, hijas o abuelas, aportan según sus posibilidades al sostenimiento económico y toda la unidad familiar es tenida en cuenta para la toma de decisiones. Además, la autoridad ya no está centralizada en el varón como proveedor económico de la familia, sino que las decisiones son concertadas con los demás miembros de esta. Esto se debe al papel que cumplen los cuidadores, y además a las normas y reglas que se consolidaron durante la ausencia del migrante y que ahora son compartidas y respetadas por todos los miembros.

Estos cambios ratifican lo expuesto por Guarnizo (2006) cuando define las señales de transformación en las familias que viven una experiencia migratoria. En primer lugar, se evidencian las modificaciones en las relaciones de género, cuando el padre retornado o la madre retornada asume roles que inicialmente estaban asignados al sexo opuesto. En segundo lugar, se aprecian alteraciones en las reglas familiares, es decir, las normas y pautas de comportamiento se hacen más flexibles. Como tercer elemento están los cambios en la autoridad y el poder dentro de la familia, ya que la autoridad es delegada o compartida por varios miembros de la unidad familiar, entre ellos, padres y cuidadores en el momento del retorno. En cuarto lugar, Guarnizo hace referencia al vacío de autoridad y afecto que no puede ser llenado totalmente por las comunicaciones a larga distancia, sin que esto signifique la desestructuración de la unidad familiar, ya que como se aprecia en los discursos de los migrantes retornados las relaciones de afecto, autoridad y cuidado se mantienen durante el proceso migratorio y posterior a la finalización de este. Por último, se confirma la movilización de apoyo de parientes para el cuidado y atención de los hijos. Estos parientes en algunos casos continúan haciendo parte de la familia aun después del retorno del migrante. Además, su papel transciende el simple cuidado y atención de los hijos y pasa a convertirse en proveedor económico y figura de autoridad dentro de las familias.

En cuanto a las permanencias se destaca el hecho de que el rol del cuidador sigue estando vinculado de manera inherente a la mujer. Es así como aquellas mujeres que han emprendido el proceso de retorno tuvieron su motivación por razones familiares, es decir, para hacerse cargo de sus hijos o padres. Igual sucede cuando se delega el cuidado de los hijos de los migrantes, pues este en la mayoría de 
los casos es encargado a las abuelas. Durante el retorno la situación no cambia, si bien es cierto que algunas mujeres trabajan o se encuentran en búsqueda de empleo, finalmente el cuidado de los hijos y el mantenimiento de la vivienda recae en las abuelas.

\section{REFERENCIAS}

Alfaro, Y. e Izaguirre, L. (2012): "Migración y perspectivas de retorno, Estado de la Situación”, en: <https:/www.academia.edu/2462222/ Migraci\%C3\%B3n_y_perspectivas_de_retorno> consultado el 10 de noviembre de 2015.

AzNAR, Y. (2009): "Identidades de retorno: La experiencia migratoria y su integración en el lugar de retorno". Ponencia presentada en el congreso de la asociación de estudios latinoamericanos, Rio de Janeiro, Brasil, 11 al 14 de junio de 2009.

BorJas, G. y Bratsber, B. (1996): "Who leaves? The outmigration of the foreign-born", en <http://www.hks.harvard.edu/fs/gborjas/publications/ journal/RESTAT1996.pdf>, consultado el 10 de noviembre de 2015.

Bryceson, D. \& Vuorela, U. (2002): The transnational family new European frontiers and global networks. Oxford, O: UniversityPress.

CASSARINO, J. (2004): “Theorising Return Migration: the Conceptual Approach to Return Migrants Revisited". International Journal on Multicultural Societies (IJMS), Vol. 6, No. 2., pp. 253-279, en: <http://unesdoc.unesco. org/images/0013/001385/138592E.pdf\#page $=60>$ consultado el 10 de noviembre de 2015.

Dellate, A., Doyagüez, C., Ituarte, M., Lapadjian, P. y Linares, C. (2009): “La inmensidad del mar. Parejas y Migración". Escola de Terapia FamiliarHospital de la Santa Creu i Sant Pau, Barelona, España, en: <http:// www.santpau.es/santpau/etf/webesp/LA\%20INMENSIDAD\%20DEL $\% 20$ MAR-\%20Parejas\%20y\%20Migraci\%F3n.pdf>, consultado el 5 de octubre de 2015.

De la fuente,Y. (2003): “La emigración de retorno, un fenómeno de actualidad". Alternativas. Cuadernos de Trabajo Social. N. 11, pp. 149-166 en: <http://rua.ua.es/dspace/bitstream/10045/5629/1/ALT_11_10.pdf>, consultado el 5 de octubre de 2015.

Di LEONARDo, M. (1992): The female world of cards and holidays: Women, families, and the work of kinship. In: T. Barrie \& M. Yalom (Ed.), Rethinking the family: Some feminist questions: 246-26. Boston, MA: Northeastern University Press.

Durand, J. (2004): "Ensayo teórico sobre la migración de retorno. El principio del rendimiento decreciente". Cuadernos Geográficos, 35 (20042), pp. 103-116. 
Ghiso, M., Tabares, O., Ramírez, L. y Morales,S. (2009a): Movilidad de Poblaciones y desarrollo humano. Cambios en la familia: los hilos invisibles de la migración. Fondo Editorial Fundación Universitaria Luis Amigó, Laboratorio Universitario de Estudios Sociales -LUES-, Medellín, Colombia.

Ghiso, M., Tabares, M., Ramírez, L. y Morales,S. (2009b): Movilidad de Poblaciones y desarrollo humano. Migración, familia y desarrollo. Claves teóricas y metodológicas del estudio de casos. Fondo Editorial Fundación Universitaria Luis Amigó, Laboratorio Universitario de Estudios Sociales -LUES-, Medellín, Colombia.

GonzÁlez de la Rocha, M. (2002): Género, Edad, Familia Y Trabajo: Reestructuración De La Sociedad Urbana En México (1980, 1990, 2000), organización doméstica y familiar en la sociedad urbana mexicana. Centro de Investigaciones y Estudios Superiores en Antropología Social, México.

GonzÁlez, L. y Restrepo, J. (2010): “Prácticas de continuidad de los vínculos parentales en las familias transnacionales colombianas en España (Comunidad Valenciana, España - eje cafetero, Colombia)", Revista Latinoamericana de Estudios de Familia. Vol. 2, enero - diciembre, 2010. pp. 79-97, en: <http:/vip.ucaldas.edu.co/revlatinofamilia/downloads/ Rlef2_4.pdf>, consultado el 20 de octubre de 2015.

GuARNIZO, L. (2006): Migración, globalización y sociedad: teorías y tendencias en el siglo XX. Ardila Gerardo (ed.). Colombia: Migraciones, transnacionalismo y desplazamiento. Cátedra Manuel Ancizar. Bogotá. Facultad de Ciencias Humanas. UN. Colección CES.

GutiérRez de PinedA, V. (1999): "La dotación cualitativa de los géneros para su status-función”, Revista Nómadas, N. 11, Octubre, 1999, pp. 148-163. Universidad Central, en: <http://www.redalyc.org/pdf/1051/ 105114277014.pdf>, consultado el 10 de septiembre de 2015.

Instituto Colombiano de Bienestar Familiar, ICBF. (2012): Caracterización de las familias en Colombia, en: <http://www.icbf.gov.co/portal/ page/portal/IntranetICBF/macroprocesos/misionales/familias/i/ Caracterizaci $\%$ C3\%B3n\%20de\%20Familias\%20(Doc\%20Final-Rev\%20 $\% 20 A C T U A L I Z A C I O N \% 20$ MA.pdf>, consultado el 20 de septiembre de 2015.

Kanaiaupuni, M. (1999): Reframing the Migration Question: An Empirical Analysis of Men, Women, and Gender in Mexico. Center for Demography and Ecology, Department of Sociology, University of Wisconsin-Madison, en: https://www.ssc.wisc.edu/cde/cdewp/99-15.pdf $>$, consultado el $10 \mathrm{de}$ noviembre de 2015.

Massey, S., Arango, J., Graeme, H., Kouaduci, A., Pellegrino, A. and Taylor, J.. (1993): "Theories of International Migration: A Review and Appraisal". Population and Development Review, Vol. 19, No. 3 (Sep., 1993), pp. 431466. Population Council. Stable URL: http://www.jstor.org/stable/2938462 Mestries, F. (2013): "Los migrantes de retorno ante un futuro incierto", Revista Sociológica, año 28, número 78, enero-abril de 2013, pp. 171-212, 
en: <http://www.revistasociologica.com.mx/pdf/7806.pdf>, consultado el 10 de noviembre de 2015.

MEJía, W. (2011): Panorama del retorno reciente de migrantes internacionales a Colombia. Documento presentado en la VI jornada Fundación Carolina: Migración y desarrollo humano, llevada a cabo en el marco de la cátedra Europa. Universidad del Norte, Barranquilla.

Mejía, W., Arrúa, E. y Bruno, S. (2015): El retorno voluntario desde España. Estudios de caso: Bolivia, Colombia y Paraguay. Programa de retorno voluntario de asistencia social cofinanciado por el Fondo Europeo para el Retorno. Organización Internacional de las Migraciones OIM. Madrid, España.

Migliorini, L., Cardinali, P. y Rania, N. (2011): "La cotidianidad de lo familiar y las habilidades de los niños". Revista Psicoperspectivas, Individuo y Sociedad. Vol. 10, No 2, pp. 183-201, en: <http://www.scielo.cl/pdf/psicop/ v10n2/art09.pdf>, consultado el 10 de noviembre de 2015.

Morad, M., Bonilla, G. y Rodríguez, M. (2011): "Vida familiar, vínculos parentales y migración transnacional colombiana: cambios y permanencias". Revista Confluenze, Vol. 3, No. 1, pp. 62-82.

Mummert, G. (1996): Mujeres de migrantes y mujeres migrantes de Michoacán: nuevos papeles para las que se quedan y las que se van. En Thomas Calvo y Gustavo López (Coordinadores). Movimientos de Población en el Occidente de México. El Colegio de Michoacán/CEMCA.

Nyberg, N. (2008): La familia transnacional de latinoamericanos en Europa en Herrera, Guioconda - Ramirez, Jacques (coords.) América Latina migrante: Estado, familias, identidades. Quito, FLACSO-Ecuador, Ministerio de Cultura del Ecuador.

Ospina, M. y Vanderbilt, A. (2009): Rol del Padre en Familias cuya madre ha migrado al exterior. Centro de Investigaciones Universidad Católica Popular del Risaralda. Pereira. Colombia.

Palacio, M. (2003): “El escenario familiar. La convergencia del conflicto armado y el desplazamiento forzado. Una lectura desde la realidad del departamento de Caldas", Universidad de Caldas, en: <http://www.bdigital.unal.edu. co/1363/16/15CAPI14.pdf>, consultado el 8 de noviembre de 2015.

Hondagneu, P. and Messner, M. (1994): Gender Displays and Men's Power: The 'New Man' and the Mexican Immigrant Man. In Harry Brod and Michael Kaufman, eds., Theorizing Masculinities. Sage Publications.

Puyana, Y. (Comp). (2003): Padres y Madres en Cinco Ciudades Colombianas Cambios y Permanencias. Almudena Editores. Bogotá.

Puyana,Y., МотоA, A., Viviel, A. (2009): Entre aquí y allá. Las familias colombianas transnacionales. Bogotá. Fundación Esperanza.

Ramirez, C. y Mendoza, L. (2013): Perfil migratorio de Colombia 2012. Bogotá,Organización Internacional para las Migraciones OIM-Colombia.

Restrepo, J. (2011): "El genograma y su aplicación como herramienta de estudio de las cadenas migratorias”. Revista Estudios Sociales, Nueva Época. Año 6, No. 7. pp. 123-150. 
Rivas, A. (2008): El papel de las remesas económicas y sociales dentro de las familias migrantes trasnacionales. Universidad Complutense de Madrid, (España) Universidad Autónoma de Madrid (España), Instituto José Ortega y Gasset (España), Universidad de Antioquia (Colombia), Universidad del Valle (Colombia).

STARK, O. (1991): The Migration of Labor. Cambridge: Basil Blackwell

Valero-Matas, J. Coca, J. y Valero-Oteo, I. (2014): “Análisis de la inmigración en España y la crisis económica”. Revista Papeles de Población, Vol. 20 No. 80, pp. 9-45, en: <http://www.redalyc.org/articulo.oa?id=11231067002>, consultado el 2 de noviembre de 2015. 